\title{
LE NUOVE SOPRINTENDENZE E IL PRINCIPIO RICOGNITIVO DELLA TUTELA
}

\author{
ANTONELLA RANALDI (*)
}

SunTO. - L'autrice si interroga su quale sarà il futuro delle Soprintendenze, vedendo nell'unificazione dei settori specialistici archeologia, belle arti e paesaggio la possibilità di invigorire e rinsaldare il sistema nazionale delle Soprintendenze. Pone l'attenzione sul principio ricognitivo della tutela, attestandosi su posizioni che ribadiscono la prevalenza nell'individuazione dei beni culturali del riconoscimento del valore in sé. Il valore è quello percepito? Come alcuni di recente sostengono favorevoli a modelli più partecipativi. O è quello riconosciuto? Ovvero, risultato di una operazione ricognitiva che spetta, pur nella discrezionalità in sé insita, agli specialisti e agli organi preposti di tutela, chiamati anche a rappresentare l'interesse percepito dalle comunità. In questa disputa di ampio respiro si evidenziano anche i rischi di tipo giuridico che la posizione soggettiva del valore percepito porta con sé. La materia complessa dei beni culturali, certe volte conflittuale nell'anteporre interessi diversi, gode di una eredità più che centenaria, che viene dalle norme di tutela nazionale con Giovanni Rosadi che nella legge 364 del 1909 chiamava l'oggetto della tutela con la parola "cose". Si era preferito il termine "cose" a quello di monumenti, immobili, oggetti mobili, perché più permeabile a comprendere quei distinti significati insieme ad altri ancora che in quelle voci non erano tutti compresi. Apriva cioè ad altri significati, che in quel momento potevano non essere ancora percepiti, ma potevano diventarlo in futuro, come appunto è avvenuto grazie alla lungimiranza di quella concezione evolutiva e aperta delle cose d'interesse, rimasta immutata anche nella terminologia dell'attuale disciplina di tutela. Conclude col affermare il valore culturale dell'individuazione delle cose da tutelare, non solo per vietare, ma per indirizzare e accendere energie.

$* * *$

(*) Soprintendente Archeologia, Belle Arti e Paesaggio per la città metropolitana di Milano, Italia. E-mail: antonella.ranaldi@beniculturali.it 
ABSTRACT. - The author asks herself about the future of the Superintendences, looking at the unification of the specialised fields (archeology, fine arts and landscape) as a mean to strengthen the national system of Superintendences. She focuses on the principle of protection reaffirming the predominance of the recognition of the value in itself in the identification of the cultural heritage. Is the value the one which is perceived, as someone recently claims in favour of more participative models? Or is the one which is recognized, in other words the result of an operation up to the specialists and the bodies responsible for the protection entitled to represent the community's interest? In this wide-ranging debate the author also highlights the legal risks that the subjective position of the perceived value brings with it. The complex matter of the cultural heritage, sometime controversial in giving preference to different interests, benefits of an ultra-centennial tradition, going back to the national protection rules by Giovanni Rosadi, who in the act $\mathrm{n}$. 364 of 1909 used to call the objects protected with the word "things". The term "thing" had been preferred to "monuments", "properties", "movables", as more opened to include the above meanings and any other which were not included: in those items it was therefere open to further meanings, not perceived at that moment, but possibly to be discerned in the future, as it happened thanks to the far-sightedness of the progressive attitude towards the "matters of interest", that didn't change in the present protection regulation. The author confirms in conclusion the cultural value of the identifications of the objects to be protected, not only to forbiel, but to direct and spur energies.

Ho partecipato a questo convegno portando i saluti del Ministro Dario Franceschini; richiamando, quindi, le novità recentissime dell'ultima riorganizzazione del Ministero che con DM del 23 gennaio 2016 introduce le soprintendenze uniche ${ }^{1}$.

L'azione del Ministero in questi ultimi due anni è stata propulsiva ed innovativa con importanti e sostanziali risultati². Si è posta l'attenzione al patrimonio culturale come risorsa capace di mettere in moto dinamiche economiche. $\mathrm{E}$ in questo senso, il Ministero ha anche acquisito la competenza sul turismo, assumendo il nome di Ministero dei beni e delle attività culturali e del turismo, nell'acronimo dal suono un po' duro di MiBACT. Dopo anni di tagli drastici, obbligati dalla crisi economica e dal

1 Invitata a partecipare a questi atti, scrivo dopo la conferma della mia nomina a soprintendente a Milano (30/6/2016), alla guida di una soprintendenza diversa da quella che avevo assunto a marzo 2015, che accorpa l'archeologia, e riduce il territorio di competenza alla città metropolitana di Milano, in luogo delle nove province lombarde della precedente Soprintendenza belle arti e paesaggio per le province di Milano, Bergamo, Como, Lecco, Lodi, Monza-Brianza, Pavia, Sondrio, Varese.

2 Vedi L. Casini, Ereditare il futuro. Dilemmi sul patrimonio culturale, Bologna 2016; rappresenta, invece, una posizione cautamente critica R. Cecchi, Abecedario. Come proteggere e valorizzare il patrimonio culturale italiano, Ginevra-Milano 2015, portando la sua esperienza nel Ministero ante riforma. 
disavanzo del debito pubblico, il Ministero ha ottenuto finanziamenti consistenti per opere di restauro sui nostri monumenti, ad esempio in Lombardia per la Certosa di Pavia, in controtendenza, e diversamente da quanto avviene in altri settori pur sofferenti. Il Ministro Dario Franceschini non si è fermato ad annunciare propositi, ha invece messo in atto un profondo rinnovamento, che trova nell'ingresso di nuove risorse umane una possibilità concreta di rafforzare i musei e le funzioni della tutela. Sono stati avviati concorsi, nominati i Direttori, per lo più stranieri, fatto che ha generato un po' di frustrazione nei ruoli del Ministero, a capo dei più prestigiosi Musei italiani, che hanno anche acquisito autonomia di spesa. Assumerà nel 2017 nuovi funzionari tecnici, archeologi, architetti, restauratori e storici dell'arte. C'è stato nel 2014 l'Artbonus ${ }^{3}$, accompagnato da una visione di corresponsabilità e compartecipazione dei privati in cambio di benefici fiscali. Il ritorno degli introiti dei biglietti ai musei stessi che li hanno prodotti ha liberato risorse che possono essere risolutive e spinge a fare meglio per avere di più. L'autonomia di spesa per i musei mira a questa stessa logica. Il modello della dirigenza ai musei non per assunzione a tempo indeterminato nei ruoli del Ministero, ma a contratto, è una spinta che sprona il Direttore nominato a raggiungere $\mathrm{i}$ risultati nei tempi di durata dell'incarico. Dalla fine dello scorso millennio, quando il Ministro Veltroni aveva captato risorse dal gioco del Lotto, bandito concorsi e assunto nel 2000 nuovo personale, non c'era stato nulla di simile, se non le assunzioni del personale nel 2010.

In questo quadro dinamico e soprattutto di importanti boccate di ossigeno (tranne che per il funzionamento), si colloca la riorganizzazione in atto che ha investito le soprintendenze, sia nell'organizzazione, sia nelle competenze, generando preoccupazioni, da una parte, nuovi stimoli e prospettive, dall'altra.

La riorganizzazione era attesa già da diversi anni. Se ne parlava all'interno del Ministero ed una commissione di esperti aveva lavorato su questo argomento già nel 2012-2013. È inutile nasconderlo, alcune avvisaglie non lasciavano presagire nulla di buono per le ormai centenarie Soprintendenze, a volte difese, ma più spesso attaccate, soprattutto ad personam. La sensazione era che fosse a rischio la loro stessa sopravvivenza. Mi ricordo bene l'attacco, a firma del noto giornalista Giovanni Valentini, sulla prima pagina di «Repubblica» Tutti $i$ no delle

3 D.L. n. 83/2014, convertito in legge n. 106 del 2014. 
Soprintendenze che rovinano $i$ tesori d'Italia (9/3/2014); con parole forti e inaccettabili sfiorava il paradosso, ma i titoli di giornale, si sa, amplificano un sentire comune, per richiamare l'attenzione. Su quella polemica di episodici detrattori, poi ritornati indietro, intervennero associazioni e intellettuali in aiuto alle Soprintendenze. Rispondeva Valentini a Quelli che difendono le Soprintendenze («Repubblica»12/3/2014) ridimensionando il tenore delle sue parole e delle sue critiche, ma il dado era stato tratto. E bene rispose l'ex Ministro Massimo Bray in un suo messaggio su face book:

«Il primo soprintendente della nostra storia fu Raffaello, che in una celebre e coraggiosa lettera del 1519 non ebbe paura a ricordare al papa Leone $\mathrm{X}$ che non doveva essere tra i suoi ultimi pensieri quello di aver cura di ciò che restava dell'antichità di Roma. Un filo diretto unisce Raffaello ai funzionari delle nostre soprintendenze: i quali lavorano per nostro conto ma soprattutto a beneficio di chi verrà dopo di noi».

Nel 2010, allora neosoprintendente, partecipai ad un corso organizzato dal Ministero, teso a darci un inquadramento più manageriale $\mathrm{e}$ aziendale, in effetti, un aspetto sul quale eravamo per formazione più carenti. I docenti, spesso provenienti da ambiti bocconiani, spiegavano la teoria degli obiettivi, della misurazione delle attività, dei risultati. Anche negli Uffici pubblici dovevano svilupparsi modelli aziendali. Uno dei risultati da perseguire era il gradimento e la soddisfazione degli utenti. La misurazione di questo esprimeva la validità dell'operato. Ecco, ciò va sicuramente bene per tanti servizi pubblici, come la scuola, gli ospedali. Il ruolo del soprintendente può invece confliggere con interessi particolari, soprattutto economici, di sfruttamento e trasformazione del paesaggio, di riutilizzo di edifici tutelati. Si è chiamati, in nome dell'art. 9 della Costituzione, a tutelare il paesaggio e il patrimonio storico artistico della nazione, applicando una normativa bene impostata che vieta le distruzioni, e sottopone ad autorizzazione del Soprintendente ogni intervento, di qualsiasi specie, sui beni tutelati. Il nostro servizio va a beneficio della conservazione e verso la trasmissione di un patrimonio ereditato da trasmettere alle future generazioni. Resta difficile quindi ottenere il gradimento dell'utenza. Corre invece l'obbligo fruttuoso di leale collaborazione con le amministrazioni, dipanare le criticità offrendo soluzioni per il loro superamento.

Mi viene da porre la domanda su quale sarà il futuro delle Soprintendenze. Invise alla totalità degli schieramenti politici, vengono 
spesso additate come un ostacolo allo sviluppo. E non deve essere stato facile al nostro Ministro convincere gli altri sulla nostra utilità, al prezzo di una riformulazione organizzativa del centro e della periferia. Con il DM del 23 gennaio 2016, si attua la seconda fase della riforma avviata con il DPCM n. 171/2014. Le soprintendenze specialistiche divise nei tre settori, beni archeologici, architettonici e paesaggistici, storico artistici ed etnoantropologici, perdono la gestione dei siti e dei musei aperti al pubblico; nascono le Soprintendenze uniche, Archeologia, belle arti e paesaggio. L'operazione di accorpamento si accompagna ad una ridefinizione, in alcuni casi, degli ambiti territoriali. Particolarmente toccata è stata la Lombardia che vede nascere quattro nuove Soprintendenze uniche, in luogo delle tre precedenti, di cui una era quella all'archeologia sull'intera regione, mentre le altre due, belle arti e paesaggio si dividevano la Lombardia, con nove province da una parte e tre dall'altra. Il vantaggio più evidente và a beneficio dell'utenza, a cui si offre un interlocutore unico per qualsiasi tipologia di beni, che siano archeologici, architettonici, paesaggistici o beni mobili d'interesse storico artistico. Favorevole è anche il ridimensionamento del territorio che vede per volontà di Dario Franceschini crearsi una Soprintendenza per la città metropolitana di Milano, a riconoscere il ruolo di una città che sta assumendo sempre di più un ruolo trainante, oltre che per l'economia, per la cultura e per modelli di gestione e amministrazione. Con la riforma viene a rafforzarsi il ruolo della tutela, almeno è nei suoi obiettivi, e più esplicite competenze nella formazione e nella ricerca.

Anche l'individuazione dei beni soggetti alla disciplina di tutela tema di questo convegno - è stata riformata, rendendo operativa l'aspirazione ad una visione 'olistica' nella Commissione al patrimonio (introdotta all'art. 33 del DPCM 171/2014) che vede riuniti i vari Soprintendenti e il Segretario regionale nel valutare i requisiti di culturalità dei beni da sottoporre a tutela. Anche le stesse Soprintendenze uniche sono figlie di questa concezione coniata 'olistica' che mira ad armonizzare e coniugare visioni e prospettive che vengono da settori diversi ${ }^{4}$.

$\mathrm{Va}$ bene l'armonizzazione, ma credo anche che la competenza tecnica specialistica vada difesa negli organici e nella dirigenza. Contraddistingue l'operato delle Soprintendenze e stabilisce quella

4 G. Volpe, Patrimonio al futuro. Un manifesto per $i$ beni culturali e paesaggistici, con la prefazione del Ministro Dario Franceschini, Milano 2015. 
distanza di giudizio richiesta per riconoscere i valori della tutela. Dall'unificazione dei settori archeologia, architettura, arte, paesaggio possono scaturire nuove possibilità nella gestione delle trasformazioni, anche nella ricerca e nella formazione, in un sistema nazionale delle Soprintendenze, che possono così meglio cogliere l'intreccio della tutela in quelle zone di confine tra settori diversi. Rimango sconcertata, invece, quando sento parlare di "casta" ${ }^{\text {, }}$ una parola che corre sulla bocca, e che scritta ancora di più offende chi lavora per le Soprintendenze. Si sostiene anche che l'interesse è quello percepito, soprattutto nel valore d'uso. Cittadini, associazioni, mezzi di comunicazione hanno sicuramente un ruolo. Ma altra cosa è la tutela, che è strumento amministrativo e comporta effetti giuridici limitativi ed obblighi sulla proprietà dei beni culturali. Si consideri che uno dei principi di chi opera in seno alle pubbliche amministrazioni è che il giudizio e l'azione non siano condizionati dall'esterno, ma siano frutto di istruttorie imparziali, in attuazione dell'art. 97 della Costituzione. Si interviene avendone titolo e nei confini e termini delineati dalla legge. Può dirsi casta? È vero però che in una materia dove la discrezionalità del giudizio è molto forte e condizionata culturalmente, possono esserci aberrazioni, laddove le motivazioni sono labili o strumentali, oppure quando i tempi dei procedimenti non vengono rispettati. Quella dei beni culturali è una materia complessa, dove gli interessi in gioco, anche di pubblica utilità, possono essere conflittuali, come tra conservazione e fruizione. Anche i veti alla circolazione delle opere fuori dal territorio nazionale, possono inibire la valorizzazione e la notorietà di artisti italiani che meriterebbero un loro riconoscimento internazionale.

La disciplina di tutela, nella sua eredità centenaria rimasta invariata e resistente ai cambiamenti, nella centralità delle cose d'interesse storico artistico, si basa sulla cognizione che il valore e quindi l'interesse sia insito nella cosa in sé, proprio e connaturato nella sua artisticità e/o storicità. Una volta vincolate, le cose d'interesse acquistano lo status di cose protette, diventando parte del patrimonio culturale da trasmettere alle generazioni future. Ciò si fonda sul riconoscimento dei valori di cui quelle cose sono depositarie in sé. La dichiarazione d'interesse viene resa oggettiva, seppure discrezionale, nell'operazione del riconoscimento, inteso in senso puramente ricognitivo e conoscitivo, riducendone quindi l'apporto soggettivo e la tentazione di enfatizzarne il soggettivi-

5 D. Manacorda, L'Italia agli Italiani. Ostruzioni per i beni culturali, Bari 2014. 
smo, nella capacità e negli strumenti e acquisizioni culturali, variabili nel tempo, di chi è chiamato nelle proprie competenze e per missione istituzionale a discernere tra cose da tutelare e non tutelare. L'interesse non è quello percepito, soggettivo, ma quello riconosciuto. Il quando e il come dipende dagli strumenti, dal bagaglio culturale e dall'occhio allenato dal mestiere che si svolge di storico, archeologo, storico dell'arte e dell'architettura, archivista, nella specificità dei valori che si vanno ad estrapolare e del ruolo e competenze istituzionali. Dove rientra a pieno titolo la percezione nel senso comune e diffuso.

In senso proprio, Cesare Brandi fondava il restauro critico nel momento metodologico del riconoscimento dell'opera d'arte' ${ }^{6}$ Ne derivava un'attenzione più consapevole alla materialità dell'oggetto, l'incipit della conservazione nel valore dell'autenticità che ad esempio Giovanni Carbonara riconduce al rinnovato culto delle reliquie nell'età della Controriforma per impulso di esponenti della cultura cristiana non di formazione artistica, ma letteraria ${ }^{7}$. Il processo del "riconoscimento" è fondante. Nella disputa tra interesse in sé e interesse percepito si apre un mondo, che mostra due prospettive; l'una, più attenta all'oggetto in re, attualizzato nel riconoscimento che è prossimo, ma diverso, dal percepire, più labile, in quanto si può percepire anche qualcosa che non c'è; l'altra, pur sensibile, catalizza l'attenzione su chi guar$\mathrm{da}$, ma è vicina a presunto. Non è il caso, ma potremmo scomodare le dispute all'interno della scolastica nel XII secolo tra nominalisti e realisti, la sintesi di Tommaso d'Aquino sul concetto di bellezza, e più oltre fino all'idealismo crociano. In ciò la legge di tutela porta con sé una felice tradizione nella prassi normativa, disconosciuta poi da orientamenti troppo critici e ideologici. Porta dritto alla questione dell'individuazione dei beni culturali, risultato di una operazione ricognitiva di riconoscimento di valori contenuti in sé nella "cosa" da tutelare. Diversamente, l'altro orientamento, secondo cui l'interesse è quello

6 C. Brandi, Teoria del restauro, Torino 1977 (I ed. 1963).

7 G. Carbonara, Teoria e metodi del restauro, in Trattato di restauro architettonico, diretto da G. Carbonara, Torino 1996, vol. I, pp. 3-16. Vedi anche sul concetto di autenticità e il valore sacrale, nel distinguo tra le tesi luterane, più selettive su oggetti di cui era certa l'autenticità, e quelle della Controriforma, che consideravano invece il valore di culto dato dalla tradizione, C. Varagnoli, Metamorfosi degli dei, metamorfosi del restauro, in Memoria e restauro dell'architettura. Saggi in onore di Salvatore Boscarino, a cura di M. Dalla Costa e G. Carbonara, Milano 2005, pp. 291-299. 
percepito, accompagna tendenze più partecipative. La mia posizione è più aderente alla prima concezione, che seguo nell'azione di tutela, non solo perché l'ho acquisita nella mia formazione, ma perché trattasi di un cardine su cui insiste la disciplina stessa di tutela, come l'abbiamo assimilata dai testi di Tommaso Alibrandi e Pier Giorgio Ferri ${ }^{8}$, che credo non possa essere liquidata, senza avere chiari gli effetti giuridici oggettivi e soggettivi che una visione e l'altra comportano.

Già nella legge di tutela del 1909, la terminologia e le questioni poste mi sembra siano state lungimiranti nel non imbrigliare la tutela in categorie troppo normate. Penso a Giovanni Rosadi, principale estensore di quella legge, la 364 del 1909, che chiamava l'oggetto della tutela con la parola "cose". Spiegava che si era preferito il termine "cose" a quello di monumenti, immobili, oggetti mobili, adottato in altre leggi e proposte perché "comprensivo di questi distinti significati e di altri ancora che forse in queste voci non sono tutti compresi". Apriva ad altri ancora significati, che in quel momento potevano essere non ancora percepiti, ma potevano diventarlo in futuro'. Come ancor più fece la Commissione Franceschini nella definizione di bene culturale come testimonianza avente valore di civiltà ${ }^{10}$. Il Codice dei beni culturali e del paesaggio (D.Lgs. 42/2004) attinge all'una e all'altra impostazione, nella terminologia e nelle "cose" di interesse culturale. Quella semplice parola era stata prescelta da Rosadi pensando alle cose delle botteghe d'arte, che non avevano l'aulicità artistica assoluta, ma diffusa e capillare della grande Officina artistica italiana. Riletta oggi, quella definizione ha permesso di ampliare sempre di più il campo delle cose tutelabili.

Vengo dalla Soprintendenza di Ravenna, e porto con me l'orgoglio di aver diretto quella che era stata nel 1897 la prima soprintendenza d'Italia ad essere istituita, con competenze snelle e operative nei vari settori, compresi gli scavi, avvalendosi di un museo da incrementare negli oggetti esposti, sulla base dei rinvenimenti effettuati nel territorio,

8 T. Alibrandi, P.G. Ferri, Il Diritto dei beni culturali, Roma 1998 (I ed. 1988).

9 A. Ranaldi, Da Rava-Rosadi a Ricci. La legge 364 del 1909. Questioni di terminologia e tutela, in Dalle 'cose di interese' ai 'beni culturali'. Ricerche e dibattiti negli Uffici MiBac dell'Emilia Romagna, a cura di P. Farinelli e P. Monari (Direzione Regionale per i Beni Culturali e Paesaggistici dell'Emilia Romagna), Bologna 2012, pp. 23-36, in particolare pp. 23-24.

10 Cfr. R. Cecchi, I beni culturali. Testimonianza materiale di civiltà, Milano 2006. 
con Corrado Ricci primo soprintendente, seguito da illustri personaggi, tra cui Giuseppe Gerola, Ambrogio Annoni, Luigi Crema. Sono ora a Milano dove forse fare tutela in un contesto più dinamico e propositivo $\mathrm{mi}$ ha portato a rivedere alcune posizioni e dogmi. Ma l'aspetto motivazionale è il primo collante che in tempi di riorganizzazione può compattare e aiuta a fare meglio.

Ritengo che anche la tutela e il lavoro delle Soprintendenze debbano assumere a pieno titolo il valore di operazioni culturali, ed in questo senso ha ragione Lorenzo Casini, tra i protagonisti di questa riforma, nel proporre in prospettiva il nome di Ministero per la Cultura e il Turismo $^{11}$. Non solo vietare, ma accendere energie, indirizzare e questa volta si partecipare.

11 L. Casini, cit. alla nota 2. 